\title{
Evaluación de la Actividad Antioxidante del Aceite Esencial Foliar de Myrcianthes leucoxyla de Norte de Santander (Colombia)
}

\author{
Clemente Granados ${ }^{(1)}$, Xiomara Yáñez ${ }^{(2)}$ y Diofanor Acevedo(1) \\ (1) Universidad de Cartagena, Facultad de Ingeniería, Programa de ingeniería de Alimentos, Avenida el \\ Consulado, Calle 30 No. 48-152. Cartagena, Bolívar-Colombia (e-mail: clementecondeg@gmail.com) \\ (2) Universidad de Pamplona, Facultad de Ciencias Básicas, Departamento de Biología y Química, \\ Extensión Villa del Rosario, Norte de Santander, Colombia. \\ *autor a quien debe ser dirigida la correspondencia
}

Recibido Sep. 16, 2013; Aceptado Nov. 12, 2013; Versión final recibida Dic. 16, 2013

\begin{abstract}
Resumen
Se ha determinado la composición química y la actividad antioxidante del aceite esencial foliar de la especie Myrcianthes leucoxyla recolectada en el departamento de Norte de Santander (Colombia). La extracción del aceite se realizó por arrastre con vapor de agua y la identificación de los componentes mayoritarios se hizo por Cromatografía de Gases de Alta Resolución. Para determinar la actividad antioxidante de los aceites se usaron dos métodos: capacidad de atrapamiento del catión radical $\mathrm{ABTS}^{+*}$ y capacidad de atrapamiento del radical DPPH. La inhibición con la metodología del radical DPPH no superó el 15\%, mientras que con el radical $\mathrm{ABTS}^{+}$el porcentaje de inhibición fue alto. Esto sugiere que en el aceite esencial estudiado se encuentran presentes compuestos donadores de hidrógeno o electrones que pueden estabilizar al radical catiónico ABTS+.
\end{abstract}

Palabras clave: aceite esencial, actividad antioxidante, Myrcianthes leucoxyla, ABTS, DPPH.

\section{Evaluation of Antioxidant Activity of the Essential Oil of Myrcianthes leucoxyla from Norte de Santander (Colombia)}

\begin{abstract}
The chemical composition and the antioxidant activity of leaf essential oil of the species Myrcianthes leucoxylain collected in the department of Norte de Santander (Colombia) were determined. The extraction of the oil was performed by steam stripping and the identification of the major components was done by High Performance Gas Chromatography. To determine the antioxidant activity of the oil two methods were employed: entrapment capacity of the radical cation ABTS+and entrapment capacity of the radical DPPH. Inhibition with the DPPH radical methodology did not exceed $15 \%$, while with the radical ABTS ${ }^{+\cdot}$ the percent inhibition was higher. This suggest that in the essential oil studied hydrogen donors or electrons which can stabilize the radical cation $\mathrm{ABTS}^{+} \cdot$ are present.
\end{abstract}

Keywords: essential oil, antioxidant activity, Myrcianthes leucoxyla, $A B T S^{+}, D P P H$. 


\section{INTRODUCCION}

Los aceites esenciales (AE) son mezclas complejas de líquidos que presentan alta volatilidad, evaporándose al contacto con el aire obtenidos a partir de diferentes partes de las plantas como flores, yemas, semillas, hojas, ramas, corteza, hierbas, madera, frutos y raíces (Acevedo et al., 2013a). Químicamente están formados por terpenos, monoterpenos y sesquiterpenos (hidrocarburos, alcoholes, cetonas, que pueden ser acíclicos, monocíclicos, bicíclicos, tricíclicos), sustancias azufradas y nitrogenadas (Muñoz et al., 2007). El valor económico de los AE y su aplicabilidad industrial están directamente relacionados con su composición química y con la actividad biológica (Stashenkoet al., 2010). El creciente interés por el uso de extractos naturales antioxidantes que puedan sustituir los aditivos sintéticos en los alimentos, resulta una alternativa prometedora para la prevención y tratamiento de enfermedades producidas por compuestos sintéticos (Olivero-Verbelet al., 2010). De hecho muchos autores han reportado propiedades antimicrobianas, antifúngicas, antioxidantes y antiradicalarias de diferentes especias y aceites esenciales y en algunos casos, una aplicación directamente relacionada con los alimentos (Acevedo et al., 2013b). La extracción de estos puede ser realizada por métodos convencionales como la destilación con arrastre de vapor (Acevedo et al., 2013a).

Dada la complejidad química de los $\mathrm{AE}$, la prueba de actividad antioxidante puede arrojar resultados diferentes dependiendo del método empleado. Por lo tanto, es aconsejable un enfoque con múltiples ensayos. Entre la lista de métodos que pueden usarse para la evaluación de la actividad antioxidante muy pocos de ellos son útiles para determinar la actividad de ambas especies (hidrofílica y lipofílica) y asegurar así una mejor comparación de los resultados (Schlesieret al., 2002). Myrcianthes leucoxyla en Colombia se conoce con el nombre común de arrayán de clima frío (Santander y Tolima), arrayán grande (Boyacá), arrayán blanco (Cundinamarca), arrayán guayabo de castilla, guayabito, o guayabo liso (Magdalena). Es un árbol de $3 \mathrm{~m}$ de altura; nativo de los Andes y distribuido en Colombia, Ecuador y Venezuela. En Colombia se encuentra en las cordilleras Central y Oriental, en los en los departamentos de Boyacá y Cundinamarca, en los municipios que conforman la Sabana de Bogotá y en el flanco occidental de la cordillera Oriental incluyendo el municipio de Pamplona (Cardozo et al., 2011; Infante-Betancouret al., 2008).

En la presente investigación se utilizaron dos métodos para determinar la capacidad antioxidante: El ensayo TEAC (por sus siglas en inglés: Trolox Equivalent Antioxidant Capacity) o ensayo del ácido 2,2-azinobis-3etil benzotioazolín-6-sulfónico (ABTS+·), el cual está basado en la captación de los antioxidantes por el radical catión $\mathrm{ABTS}^{+\cdot}$ generado en el medio de reacción, usando como patrón el ácido 6-hidroxi-2,5,7,8 tetrametilcromán-2-carboxílico (Trolox), un análogo sintético hidrosoluble de la vitamina E. Este radical catión ABTS+. posee una coloración verde-azulada con un máximo de absorción a $415 \mathrm{~nm}$ (Granados et al., 2012). El segundo método se basa en la reducción del radical $\mathrm{DPPH}(2,2$-difenil-1picrilhidracilo) por los compuestos antioxidantes de la muestra del AE. El radical es estable y tiene una coloración púrpura que se pierde progresivamente cuando se añade la muestra conteniendo sustancias antioxidantes. La decoloración del radical se determina a $515 \mathrm{~nm}$ y la cuantificación se realiza por lo general, empleando soluciones patrón de Trolox(Brand-Williams et al., 1995). Se evaluó la capacidad antioxidante del AE foliar de la especie $M$. leucoxyla, conocida comúnmente como arrayán de clima frío, cuyos resultados podrían utilizarse como una alternativa en futuros estudios que busquen reemplazar los antioxidantes artificiales en alimentos.

\section{MATERIALES Y MÉTODOS}

\section{Selección de la Materia Prima Vegetal}

La especie conocida comúnmente como arrayán de clima frío (M. leucoxyla) fue recolectada en el municipio de Pamplona, en la vereda de los Animes en la carretera que conduce de Pamplona a Bucaramanga. Se tomaron muestras pertenecientes al material foliar de las plantas adultas, hojas enteras y verdes. Las hojas fueron recolectadas en horas de la tarde y en época de invierno. Un ejemplar fue colectado por el botánico Luis Roberto Sánchez Montaño y reposa en el Herbario Regional Catatumbo-Sarare de la Universidad de Pamplona.

\section{Extracción del $A E$}

La obtención del $A E$ se realizó por destilación con arrastre de vapor. Se empleó un equipo con manta de calentamiento para un balón de 5 litros (L). Se tomaron $400 \mathrm{~g}$ de material vegetal previamente identificado, seleccionado y pesado, los cuales fueron introducidos en un balón, al cual se adicionó $1 \mathrm{~L}$ de agua. El tiempo de extracción fue de dos horas y media. EI AE se colectó en un recipiente tipo Dean Stark. Este se separó por decantación, fue medido con jeringa de $2 \mathrm{~mL}$ para establecer el rendimiento y almacenado en un vial de $4 \mathrm{~mL}$ de vidrio color ámbar (Stashenkoet al., 2010). El rendimiento del AE obtenido se calculó mediante la Ecuación 1: 
$\%$ Rendimiento $=\frac{(\mathrm{mL} \text { de aceite esencial })}{\text { Peso en gramos del material vegetal }} \times 100 \%$

\section{Determinación de propiedades físicas del $A E$}

Al $\mathrm{AE}$ obtenido se le determinó densidad relativa a $20^{\circ} \mathrm{C}$ en picnómetro de $2 \mathrm{~mL}$; rotación óptica en polarímetro; índice de refracción en Refractómetro ABBE; punto de ebullición en tubo de Thiele y solubilidad en etanol. Para este último se emplearon soluciones etanólicas de 20, 40, 60 y $80 \%$ v/v. Tomando $100 \mu \mathrm{L}$ de cada solución, se agregaron lentamente pequeños volúmenes del $A E(2 \mu L)$, hasta que la solución se tornó turbia (punto de saturación). Cada determinación se hizo por duplicado.

\section{Análisis de los componentes mayoritarios del $A E$}

Se realizó por Cromatografía de Gases de Alta Resolución (CGAR). El equipo utilizado fue un CGAR HP6890 Series, en interfase con un detector selectivo de masas HP5973 Network conectado en línea con un sistema HP-MS ChemStation y la base de datos NIST-2005. Se tomaron $30 \mu \mathrm{L}$ del AE y se disolvieron en $1 \mathrm{~mL}$ de diclorometano. Se inyectó $1 \mu \mathrm{L}$ en modo split (10:1). Los espectros de masas (EM) se obtuvieron por ionización electrónica con barrido automático. Cada EM fue comparado con los estándares de la base de datos y los datos reportados en la literatura (Adams, 2005). Para obtener los índices de Kováts (IK) relativos a los patrones de alcanos $\mathrm{C}_{8}-\mathrm{C}_{32}$, el análisis se realizó en un CGAR HP 6890A Series II con detector FID a $250^{\circ} \mathrm{C}$. Para la identificación y cuantificación de los componentes de cada AE se utilizó el criterio cromatográfico (IK experimentales y teóricos) y el criterio espectral (EM). La composición porcentual por CG-FID de cada componente se analizó por el método de normalización de áreas (Stashenko, 2010).

\section{Evaluación de la actividad antioxidante de cada $A E$}

Se usaron dos métodos: Capacidad de atrapamiento del catión radical ABTS+.y del radical DPPH: De forma exploratoria, se realizaron ensayos preliminares a concentraciones entre 300 y 100 ppm para cada muestra de $A E$ y de acuerdo con los resultados obtenidos se evaluó la capacidad antioxidante a concentraciones entre 1 y 100 ppm. Para ambos métodos se usó como estándar Trolox, cuya capacidad antioxidante se evaluó a las mismas condiciones de trabajo de las muestras. Los resultados son expresados como capacidad antioxidante equivalente al Trolox (TEAC; $\mu$ mol de Trolox/gramos de sustancia evaluada) y capacidad antioxidante equivalente al ácido ascórbico (AAEAC; $\mu$ mol de ácido ascórbico/gramos de sustancia evaluada).Todos los ensayos se realizaron por triplicado.

\section{Método del radical DPPH}

Se empleó el método de Brand-Williams et al., 1995 con algunas modificaciones, $40 \mu \mathrm{L}$ de muestra fueron adicionados a $960 \mu \mathrm{L}$ de una solución metanólica de DPPH, y se evaluó la capacidad de las muestras para atrapar el radical DPPH, por medio de la disminución en la absorbancia leída luego de 30 minutos de reacción a una longitud de onda de $517 \mathrm{~nm}$, y se comparó el valor con la curva de referencia construida con Trolox como patrón primario, expresando los resultados como valores TEAC ( $\mu$ mol Trolox/100g de muestra).

La absorbancia se midió en un espectrofotómetro Genesis 20 ThermoSpectronic modelo 4001/4, y se calculó el porcentaje de inhibición (\% Inh) usando la Ecuación (2). Siendo A Muestra la absorbancia del Aceite Esencial (AE), para lo cual se preparó una solución de 10000 ppm, 50 mg en 5ml de Dimetil sulfóxido (DMSO, Merck), y a partir de ésta, soluciones diluidas a diferentes concentraciones (1, 50, 100, 150, 200, 250 y $300 \mathrm{ppm}$ ), tomando en cada ensayo $40 \mu \mathrm{L}$ y adicionando $960 \mu \mathrm{L}$ de la solución del radical $\mathrm{DPPH}^{\circ}$. A Blancoes la absorbancia del Blanco, preparado con $40 \mu \mathrm{L}$ de la muestra y $960 \mu \mathrm{L}$ de $\mathrm{MeOH}$, y $A_{\text {Referencia }}$ es la absorbancia de la Referencia, preparada con $40 \mu \mathrm{L}$ de DMSO y $960 \mu \mathrm{L}$ de la solución del radical DPPH'(Granados, et al., 2012).

$\% \operatorname{Inh}=\left[1-\frac{A_{\text {Muestra }}-A_{\text {Blanco }}}{A_{\text {Referencia }}}\right] \times 100$

\section{Método del radical $A B T S^{+}$}

Se empleó el método de Re et al., 1999 con algunas modificaciones, $10 \mu \mathrm{L}$ de muestra fueron adicionados a $990 \mu \mathrm{L}$ de una solución de $\mathrm{ABTS}^{+}$y se evaluó la capacidad de cada muestra para atrapar el radical catiónico, por medio de la disminución en la absorbancia leída luego de 30 minutos de reacción, a una longitud de onda de 732 nm (Zapata, et al., 2013; Heras, et al., 2013). El valor de absorbancia se comparó 
con la curva de referencia construida con Trolox como estándar y los resultados se expresan como valores TEAC (TroloxEquivalentAntioxidantCapacity), $\mu \mathrm{mol}$ Trolox/100 g de muestra.

\section{ANÁLISIS DE RESULTADOS}

\section{Rendimiento del aceite esencial y propiedades físicas}

En la Tabla 1, se muestra el rendimiento obtenido en la extracción del aceite esencial foliar (AE) y las propiedades físicas medidas para la especie vegetal analizada.

Tabla 1:Rendimiento y propiedades físicas del AE de Myrcianthes leucoxyla.

\begin{tabular}{|c|c|}
\hline Análisis & Resultado \\
\hline Rendimiento del $\mathrm{AE}$ & $0,3 \%$ \\
\hline Densidad especifica $20^{\circ} \mathrm{C}$ & $0,92 \mathrm{~g} / \mathrm{mL}$ \\
\hline Índice de refracción $20^{\circ} \mathrm{C}$ & 1,4774 \\
\hline Rotación óptica $a 0^{\circ} \mathrm{C}$ & $-6^{\circ} \mathrm{a}+4^{\circ}$ \\
\hline Solubilidad en etanol & Positiva \\
\hline
\end{tabular}

El rendimiento de $\mathrm{AE}$ foliar de $M$. leucoxyla seencontró bastante bajo si se tiene en cuenta que el resultado promedio que se debe obtener, a nivel general, es entre el 1 y el 3\%.Muñozet al., (2007) reportaron en su estudio rendimientos obtenidos en orégano común de $0,1 \%$; orégano cimarrón (Lippiaoriganoides) de 2,3\%; tomillo de $0,2 \%$ y orégano de castilla de $1,6 \%$. Las densidades cercanas a $0,9 \mathrm{~g} / \mathrm{mL}$ y los índices de refracción son característicos de los AE de diversas especies (Bandoni, 2002).

\section{Componentes volátiles mayoritarios del AE de Myrcianthes leucoxyla}

De acuerdo con el perfil cromatográfico de los componentes detectados por CGAR, se relacionan los 10 componentes mayoritarios del $\mathrm{AE}$ de arrayán de clima frío ( $M$. leucoxila) con una concentración superior al $1 \%$ (Tabla 2). Se destacan los monoterpenos $\alpha$-Pineno (28,4\%) y 1,8-Cineol, syn.Eucaliptol $(15,17 \%)$, de forma similar a lo que reporta Carhuapoma (2006), sobre los componentes del aceite de Luma chequen (Mirtácea), que también presenta en mayor cantidad los monoterpenos a-Pineno $(57,1 \%), 1,8$-Cineol $(12,1 \%)$ y Linalol (5,5\%). Además el $\mathrm{AE}$ posee Cariofileno, un sesquiterpeno presente en otros $\mathrm{AE}$ con actividad antioxidante (Acevedo, et al., 2013a, 2013b; Huang, 2011).

Tabla 2: Componentes mayoritarios y concentración (\%) del AE de Myrcianthes leucoxyla

\begin{tabular}{|c|c|c|c|c|c|}
\hline No. & $t_{R}$ & Compuesto & $I_{\text {Teórico }}{ }^{(1)}$ & $I K_{\text {Exp. }}{ }^{(2)}$ & Concentración (\%)(3) \\
\hline 1 & 7,159 & a-Tuyeno & 924 & 927 & 1,60 \\
\hline 2 & 7,355 & $\alpha-P i n e n o(4)$ & 939 & 935 & 28,40 \\
\hline 3 & 8,916 & $\beta$-Mirceno & 990 & 991 & 1,71 \\
\hline 4 & 9,889 & 1,8-Cineol & 1031 & 1033 & 15,70 \\
\hline 5 & 19,797 & a-Cubebeno & 1345 & 1346 & 1,51 \\
\hline 6 & 22,607 & Z-Cariofileno & 1408 & 1415 & 3,79 \\
\hline 7 & 22,816 & $\beta$-Humuleno & 1436 & 1430 & 1,95 \\
\hline 8 & 24,787 & (-)-Spatulenol & 1577 & 1575 & 3,30 \\
\hline 9 & 24,929 & Óxido de Cariofileno & 1583 & 1590 & 3,04 \\
\hline 10 & 25,534 & Guaiol & 1600 & 1610 & 3,13 \\
\hline
\end{tabular}

El IK Teórico fue según Adams (2005), el IK Exp corresponde a los datos calculados por CGAR-FID en columna HP-5, y al final se muestra la concentración relativa, promedio de extracciones realizadas por triplicado.

\section{Evaluación de la actividad antioxidante del $A E$ :}

Resultados frente al radical DPPH: En la Tabla 3, se observa que a diferentes concentraciones entre $100 \mathrm{y}$ $300 \mathrm{ppm}$ el porcentaje de inhibición (\% Inh.) con el método del radical DPPH, en ningún caso superó el $15 \%$, razón por la cual se descartó continuar la evaluación de la actividad antioxidante con esta 
metodología, teniendo en cuenta que se esperan valores de Inhibición superiores al 30\% (Granados et al., 2012). Se podría decir que en las muestras evaluadas no se encuentran presentes compuestos que puedan estabilizar al radical DPPH· (Torres et al., 2007).

Tabla 3: Capacidad antioxidante (\% Inhibición) frente al radical DPPH de los AE.

\begin{tabular}{|c|c|c|c|c|c|}
\hline $\mathrm{AE}(\mathrm{ppm})$ & 100 & 150 & 200 & 250 & 300 \\
\hline$\%$ Inh. & 3,84 & 4,06 & 5,01 & 7,16 & 12,02 \\
\hline
\end{tabular}

Resultados frente al radical catiónico ABTS+; Al realizar ensayos preliminares frente al radical ABTS+., entre concentraciones de 150 y 300 ppm se alcanzaron valores superiores al $80 \%$ de inhibición (Ver Tabla 4). Además se observó que a 100 ppm el porcentaje de inhibición (\% Inh.) del AE de M. leucoxyla fue de 41,04, lo cual sugiere que en éste se encuentran presentes compuestos donadores de hidrógeno o de electrones que puedan estabilizar al radical catiónico $\mathrm{ABTS}^{+}$. Luego se realizaron ensayos con concentraciones menores de $\mathrm{AE}$ de 1 y $50 \mathrm{ppm}$ para determinar la concentración mínima a la cual el $\mathrm{AE}$ mantiene su capacidad antioxidante.

Tabla 4: Capacidad antioxidante (\% Inhibición) frente al ABTS+ del AE de Myrcianthes leucoxyla.

\begin{tabular}{|c|c|c|c|c|c|c|c|}
\hline $\mathrm{AE}(\mathrm{ppm})$ & 1 & 50 & 100 & 150 & 200 & 250 & 300 \\
\hline$\%$ Inh. & 2,02 & 24,06 & 41,04 & 86,60 & 87,99 & 88,00 & 89,99 \\
\hline
\end{tabular}

En el caso del AE de Minthostachysmollissólo presentó actividad antioxidante a partir de 500 ppm cuando se alcanzan porcentajes de inhibición superiores al $60 \%$, lo cual contrasta con el arrayán de clima frío, el cual comienza a tener porcentajes inhibitorios del $40 \%$ a partir de 100 ppm de AE(Granados, et al., 2012).

En general, los valores de inhibición del radical DPPH·fueron menores en comparación a los valores frente al radical $\mathrm{ABTS}^{+}$, sugiriendo que los compuestos antioxidantes presentes en el $\mathrm{AE}$ son altamente hidrofílicos siendo más sensibles a la técnica del ABTS+· (Guala, 2009; Heras, 2013; Torres, et al., 2013; Zulueta, et al., 2009).

Con la gráfica\% Inhibición del AE vs. Logaritmo $\left(\log _{10}\right)$ de la concentración fue calculado el IC 50 para el AE. El valor IC 50 $_{5}$ (concentración a la cual el \% Inh. es igual a 50) para el AE de M. leucoxylafue de 118.4 ppm. Este valor se convierte en Equivalentes Trolox (TEAC-por sus siglas en inglés, TroloxEquivalentAntioxidantCapacity), de acuerdo con la siguiente fórmula:

TEAC $=\frac{I C 50 \text { DEL Trolox }\left(\frac{\mu m o l}{m L}\right)}{\text { IC50 de la muestra }\left(\frac{g}{m L}\right)}$

El valor TEAC fue de $121.09 \mu \mathrm{mol}$ de Trolox/g de extracto, el cual es menor que el reportado para el orégano, una especie de referencia con reconocida capacidad antioxidante (Granados, et al., 2012).

\section{CONCLUSIONES}

La actividad antioxidante encontrada en el AE de $M$. leucoxyla posibilita la realización de posteriores estudios, en la búsqueda de su aplicación en la industria de alimentos como posible sustituto de los antioxidantes sintéticos, dada la potencial capacidad para atrapar radicales libres.

\section{REFERENCIAS}

Acevedo, D., M. Navarro y L. Monrroy, Composición química del aceite esencial de hojas de Orégano (Origanumvulgare), Información Tecnológica: 24(4), en prensa (2013a).

Acevedo, D., M. Navarro y L. Monrroy, Composición química del aceite esencial de las hojas de toronjil (Melissa officinalis L.), Información Tecnológica: 24(4), en prensa (2013b).

Adams, R., Identification of essential oil components by gas chromatography/mass spectroscopy, 469p, Allured Publishing Corporation: Carol Stream, Lllinois, USA (2005). 
Bandoni, A.I., Los Recursos Vegetales Aromáticos en Latinoamérica, 1a Ed., 250p, Editorial de la Universidad Nacional de la Plata, La Plata, Argentina(2002).

Brand-Williams, W., M.E. Cuvelier y C. Berset, Use of a free radical method to evaluate antioxidant activity, LebensmittelWissenchaft und Technologie: 28(1), 25-30 (1995).

Cardozo, R.H., y otros diez autores, Especies útiles en la Región Andina de Colombia. Tomo I, $1^{\text {a }}$ Ed, 179188, Imprenta Nacional de Colombia, 179-188, Bogotá, Colombia (2011).

Carhuapoma, M. y otros tres autores, Estudio de la composición química y actividad antioxidante del aceite esencial de Luma chequen (Molina) A. Gray “arrayán”, Ciencia e Investigación: 8(2), 73-79 (2005).

Granados, C., X. Yáñez, y G. Santafé, Evaluaciòn de la actividad antioxidante del aceite esencial foliar de Calycolpusmoritzianus y Minthostachysmollis, de Norte de Santander, Bistua: Revista de la Facultad de Ciencias Básicas: 10(1), 12-23 (2012).

Guala, M., Evaluación del Poder Antioxidante de Fracciones de Aceite Esencial Crudo de Schinus molle L. obtenidas por destilación al vacío, Información Tecnológica: 20(2), 83-88 (2009).

Heras, I., Alvis, A. y G. Arrazola. Optimización del Proceso de Extracción de Antocianinas y Evaluación de la Capacidad Antioxidante de Berenjena (Solana melonera L.). InformaciónTecnológica: 24 (5), 93-102 (2013).

Huang, C.A., Study of four antioxidant activities and major chemical component analysis of twenty-five commonly used essential oils, J. Cosmet. Sci.: 62(4):393-404 (2011).

Infante-Betancour, J., A. Jara-Muñoz y O. Rivera-Díaz, Árboles y arbustos más frecuentes de la Universidad Nacional de Colombia, sede Bogotá, $1^{\text {a }}$ Ed., 31p., Universidad Nacional de Colombia, Bogotá, Colombia (2008).

Muñoz, A. y otros seis autores, Composición y capacidad antioxidante de especies aromáticas y medicinales con alto contenido de Timol y Carvacrol, Scientia et Technica: 13(33), 125-128 (2007).

Olivero-Verbel, J. y otroscuatroautores, Chemical composition and antioxidant activity of essential oils isolated from Colombian plants, Rev. bras. Farmacogn: 20(4), 568-574 (2010).

Re, R., N. Pellegrini, A. Proteggente, A. Pannala, M. Yang y C. Rice-Evans, Antioxidant activity applying an improved ABTS radical cationdecolorization assay, Free Radicals in Biology and Medicine: 26(9/10), 12311237 (1999).

Schlesier, K. y otrostresautores, Assessment of antioxidant activity by using different in vitro methods, Free Rad Res.: 36(2):177-187 (2002).

Stashenko, E. y otrosseisautores, Lippiaoriganoideschemotype differentiation based on essential oil GC-MS and principal component analysis, J. Sep. Sci.: 33(1), 93-103 (2010).

Torres, O. y otros tres autores, Estudio químico y obtención de principios activos de la especie Rolliniapittieri (Annonaceae) del Alto Sinú, Scientiae et Technica, 13(133), 55-58 (2007).

Zapata, K., Cortes, F.B. y B.A., Rojano. Polifenoles y Actividad Antioxidante del Fruto de Guayaba Agria (Psidiumaraca), Información Tecnológica: 24(5), 103-112 (2013).

Zulueta, A., M.J. Esteve y A. Frígola, ORAC and TEAC assays comparison to measure the antioxidant capacity of food products, Food Chemistry: 114(1), 310-316 (2009). 